\title{
ANALYSIS OF THE EFFECT OF CERTAIN FORCINGS ON THE NONOSCILLATORY SOLUTIONS OF EVEN ORDER EQUATIONS
}

\author{
ATHANASSIOS G. KARTSATOS \\ (Received 22 April 1976) \\ Communicated by N. S. Trudinger
}

\begin{abstract}
Properties of solutions of

$$
x^{(n)}+H(t, x)=Q(t), \quad n \text { even, }
$$

are studied in the case $u H(t, u)>0$ for $u \neq 0$. It is shown that two inequalities may always be associated with (I) in such a way that if one of these inequalities has a small positive solution and the other inequality has a small negative solution, then (I) is oscillatory. Further asymptotic properties of (I) are studied under assumptions involving intermediate antiderivatives $P^{(i)}(t)$, with $P^{(n)}=Q$. Several results of this type ensure the non-existence of positive solutions of (I).
\end{abstract}

Subject classification (Amer. Math. Soc. (MOS), 1970): primary 34 C 10

\section{Introduction}

Kartsatos (1971) initiated the study of oscillation criteria concerning the solutions of forced equations of the form

$$
x^{(n)}+H(t, x)=Q(t), \quad n \text { even, }
$$

where $x H(t, x) \geqslant 0$ for all $(t, x), H$ is increasing in the second variable, the homogeneous (I) is oscillatory, and $Q$ is oscillatory and "small" in a certain sense. Only superlinear equations were considered in Kartsatos (1971), while in a later paper (Kartsatos, 1972) the author considered periodic-like and oscillating forcings $Q(t)$. Some results were also given by Teufel (1972) but for $n=2$. The above results of the author were extended by Kusano and Onose (1974), True (1975), Foster (1976a, b), Staikos and Sficas (1975a, b) and Onose (1973). Atkinson (1972/3) also extended most of the above results of the author but for $n=2$. The main oscillation result of Atkinson was shown to hold in the general case by Kartsatos (to appear) (cf. also Onose, 1975). Several results on forced or perturbed equations have also been recently obtained by Kartsatos (1976a, b, c), Kartsatos and Manougian (1974, 1976), Graef and Spikes (to appear) and Graef (to appear). For a recent survey of results on the subject the reader is referred to Kartsatos (to appear). 
The main purpose of the present paper is to study the behaviour of nonoscillatory solutions of (I). For example, two inequalities are associated with (I), and we show that if one of them has a small positive solution and the other one has a small negative solution, then (I) is oscillatory if the homogeneous (I) is oscillatory. In general, it is quite a difficult problem to find such solutions of both these inequalities. As a side-result, we obtain that if only one of these inequalities is satisfied, then the existence of one of the above kinds of nonoscillatory solutions is excluded.

We should mention now that the existence of a small positive solution of a differential equation (and hence inequality) could be suggested by physical considerations like, for example, the ones in Nehari (1963).

Several other results are also obtained depending on further properties of the forcing $Q(t)$.

\section{Preliminaries}

In what follows, $n$ will always be an even integer with $n \geqslant 4, T$ a nonnegative number, $R_{T}=[T, \infty), R=(-\infty, \infty)$ and $R_{+}=[0, \infty)$. By a solution of (I) we mean any function $x(t), t \geqslant t_{x}$, which is $n$ times continuously differentiable and satisfies (I) on the interval $\left[t_{x}, \infty\right)$. Here $t_{x} \geqslant T$ depends on the particular solution under consideration. A function $f:[a, \infty) \rightarrow R, a \geqslant T$, is said to be "oscillatory" if it has an unbounded set of zeros on $[a, \infty)$. (I) is said to be "oscillatory" if every solution of it is oscillatory. (I) is " $B$-oscillatory" if every bounded solution of it is oscillatory.

We now state an auxiliary lemma, a stronger version of which was established by Kartsatos (1975).

Lemma A. Consider the equation

$$
x^{(n)}+H(t, x)=0,
$$

and the inequality

$$
x^{(n)}+H(t, x) \leqslant 0,
$$

where $H: R_{T} \times R_{0} \rightarrow R_{+}\left(R_{0}=\left[u_{0}, \infty\right), u_{0}>0\right)$ is continuous and increasing with respect to $x$. Then the existence of a solution (bounded solution) $x(t)$ of (2.1) with $x\left(t_{1}\right)=u_{1} \geqslant u_{0}$ for some $t_{1} \geqslant T$ and $x^{\prime}(t) \geqslant 0, t \in\left[t_{1}, \infty\right)$, implies the existence of a solution (bounded solution) of $\left(\mathrm{I}_{H}\right)$ with $y\left(t_{2}\right)=u_{1}$ and $y^{\prime}(t) \geqslant 0, t \in\left[t_{2}, \infty\right)$, for some $t_{2} \geqslant t_{1}$.

Now let us show that under the assumptions of this lemma, the existence of a solution $x(t)$ of the inequality

$$
x^{(n)}+H(t, x) \geqslant 0
$$

with $x(t)<0, x^{\prime}(t) \leqslant 0$ eventually, implies the same fact for $\left(\mathrm{I}_{H}\right)$ if $H$ is sufficiently defined for negative $x$. In fact, let $u(t) \equiv-x(t), t \geqslant t_{1}$. Then $u(t)$ is a positive solution 
of the inequality

$$
u^{(n)}-H(t,-u) \leqslant 0 .
$$

However, applying Lemma $A$ to the function $-H(t,-x)$ we obtain that the equation

$$
v^{(n)}-H(t,-v)=0
$$

has a solution $v(t)$ such that $v(t)>0, v^{\prime}(t) \geqslant 0$ eventually. Letting $w(t) \equiv-v(t)$ in (2.4) we have our assertion.

In what follows the functions $Q: R_{T} \rightarrow R, H: R_{T} \times R \rightarrow R$ will be assumed continuous. Moreover, $H$ will be increasing in the second variable and such that $u H(t, u)>0$ for all $(t, u) \in R_{T} \times R$ with $u \neq 0$.

\section{Main results}

We shall say that the function $H$ satisfies Hypothesis (S) if $H(t, u)$ is strictly increasing in $u$ and such that for every $t \in R_{T}$ and every $\mu_{1}, \mu_{2} \in R$ with $\mu_{1} \mu_{2}>0$, $\left|\mu_{1}\right|<\left|\mu_{2}\right|$

$$
\left|H\left(t, \mu_{1}\right)\right| \leqslant k\left(\mu_{1}, \mu_{2}\right)\left|H\left(t, \mu_{2}\right)\right|,
$$

where $k$ is a constant depending on $\mu_{1}, \mu_{2}$ and such that $0<k\left(\mu_{1}, \mu_{2}\right)<1$.

THEOREM 3.1. Let H satisfy Hypothesis (S). Moreover, let the equation

$$
x^{(n)}+\mu H(t, x)=0
$$

be oscillatory (B-oscillatory) for every $\mu>0$.

Let the inequality

$$
x^{(n)}+H(t, x) \leqslant Q(t)
$$

have an eventually positive solution $x_{1}(t)$, and the inequality

$$
x^{(n)}+H(t, x) \geqslant Q(t)
$$

have an eventually negative solution $x_{2}(t)$ such that

$$
\lim _{t \rightarrow \infty} x_{1}(t)=\lim _{t \rightarrow \infty} x_{2}(t)=0 .
$$

Then (I) is oscillatory (B-oscillatory).

Proof. Let $x_{2}(t)<0, t \geqslant t_{1} \geqslant T$, satisfy (3.3), and let (3.1) be oscillatory. Then the function $u(t) \equiv x(t)-x_{2}(t), t \geqslant t_{1}$, for some positive solution $x(t)$ of $(I)$, satisfies

$$
u^{(n)}(t)+H\left(t, u(t)+x_{2}(t)\right)-H\left(t, x_{2}(t)\right) \leqslant 0 .
$$

Let us assume that $u(t)>0, t \geqslant t_{2} \geqslant t_{1}$. Then $u^{(n)}(t)<0$ for $t \geqslant t_{2}$, and since $n$ is even, it follows that $u^{\prime}(t)>0$ for all large $t$, say for $t \geqslant t_{3} \geqslant t_{2}$. Now choose $\varepsilon>0$ so 
that $\varepsilon<u\left(t_{3}\right) / 2$, and $\left|x_{2}(t)\right|<\varepsilon$ for every $t \geqslant$ (some) $t_{4} \geqslant t_{3}$. Then from (3.5) we obtain

$$
\begin{aligned}
u^{(n)}(t)+ & H(t, u(t)-\varepsilon)-H(t, \varepsilon) \\
& \leqslant u^{(n)}(t)+H\left(t, u(t)+x_{2}(t)\right)-H\left(t, x_{2}(t)\right) \leqslant 0, \quad t \geqslant t_{4} .
\end{aligned}
$$

Moreover, $u(t)-2 \varepsilon>u\left(t_{3}\right)-2 \varepsilon>0$ for all $t \geqslant t_{4}$. Consequently,

$$
H(t, u(t)-\varepsilon)-H(t, \varepsilon)>0, \quad t \geqslant t_{4} .
$$

Now letting $v(t) \equiv u(t)-\varepsilon$ in (3.6) we obtain that $v(t), t \geqslant t_{4}$, is a positive solution to the inequality

$$
v^{(n)}+H(t, v)-H(t, \varepsilon) \leqslant 0, \quad t \geqslant t_{4},
$$

and such that $v^{\prime}(t)>0, t \geqslant t_{4}$ and $v(t)>\varepsilon$ for every $t \geqslant t_{4}$. From Lemma $\mathrm{A}$ it follows now that this is also true (say for $t \geqslant t_{4}$ ) for the equation

$$
z^{(n)}+H(t, z)-H(t, \varepsilon)=0 .
$$

Let $z(t)$ be such a solution of (3.9) with $z(t), z^{\prime}(t)>0$ for $t \geqslant t_{4}$ and $z\left(t_{4}\right)>\varepsilon$. Then, since $H(t, x)$ is strictly increasing in $x$, letting $\mu=1-k\left(\varepsilon, z\left(t_{4}\right)\right)<1$ we obtain that the equation

$$
w^{(n)}+[1-[H(t, \varepsilon) / H(t, z(t))]] H(t, w)=0
$$

has a positive solution $z(t), t \geqslant t_{4}$, with the coefficient of $H(t, w(t))$ bounded below by the constant $\mu$. Since (3.1) is oscillatory for every $\mu \geqslant 0$, an application of the comparison theorem in Kartsatos (1975), Theorem 2.1 (cf. also Kartsatos, to appear) yields that (3.10) must also be oscillatory, which is a contradiction. It follows that there exists a sequence $\left\{t_{m}\right\}, m=1,2, \ldots$, such that $\lim _{m \rightarrow \infty} t_{m}=\infty$ and $x\left(t_{m}\right)<x_{2}\left(t_{m}\right)<0, m=1,2, \ldots$, which contradicts the positiveness of $x(t)$. A similar proof covers the case of a positive solution $x_{1}(t)$, and the case of (3.1) being $B$-oscillatory. We omit these arguments.

As pointed out in the introduction, it is usually difficult to find both functions $x_{1}(t), x_{2}(t)$. However, finding only one of these functions is a much easier task, and this would of course ensure the nonexistence of positive or negative solutions of (I). Let us consider an example to illustrate this last assertion:

$$
x^{(4 m)}+t^{-\alpha}|x|^{\beta} \operatorname{sgn} x=\left(\frac{1}{2}+\sin t\right) t^{-\gamma} .
$$

Here $m$ is a positive integer, $1<\alpha<4 m-1, \beta>1$ and $1<\gamma<4 m+1$. It turns out that the function

$$
x_{1}(t)=(2+\sin t) t^{-\gamma}
$$

satisfies the inequality

$$
\left.x_{1}^{(4 m)}(t)+t^{-\alpha} x_{1}^{\beta}(t) \leqslant \frac{1}{2}+\sin t\right) t^{-\beta} \text { for all large } t .
$$

Thus (3.11) does not have any negative solutions.

The oscillation of (3.1) here follows from known results (cf., for example Kartsatos, 1971). 
From the proof of the above theorem we can obtain now some other results which we formulate here as corollaries but only for positive solutions of (I), leaving to the reader the dual statements for negative solutions.

Corollary 3.1. Let the assumptions on $H, Q$ of Theorem 3.1 be satisfied. Let $x_{1}(t)$ be a solution of (3.2) with $\lim _{t \rightarrow \infty} x_{1}(t)=0$. Then every eventually positive (bounded and positive) solution $x(t)$ of (I) satisfies $\lim _{t \rightarrow \infty} \inf x(t)=0$ if (3.1) is oscillatory (B-oscillatory) for every $\mu>0$.

Corollary 3.2. Let the assumptions on $H, Q$ of Theorem (3.1) be satisfied. Let $x_{1}(t)$ be an eventually positive solution of $(\mathrm{I})$ with $\lim _{t \rightarrow \infty} x_{1}(t)=0$. Assume that $x_{2}(t)$ is another solution of (I) with the same property. Then $x_{1}(t)-x_{2}(t)$ is an oscillatory function provided that (3.1) is B-oscillatory for every $\mu>0$.

The reader is referred to the paper of Kartsatos (1976b) where conditions are imposed on $H, Q$ so that the above function $x_{1}(t)-x_{2}(t)$ is identically equal to zero for all large $t$. The conclusion there is that (I) can have at most one positive solution.

In the following two theorems upper or lower appraisals are obtained for positive solutions of (I). The reader should keep in mind that, under dual hypotheses, similar results hold for the negative solutions.

TheOREM 3.2. Let $P: R_{T} \rightarrow R_{+}$be continuous and such that $P^{(j)}(t) \equiv Q(t)$, where $j$ is an odd positive integer with $1 \leqslant j \leqslant n-1$. Moreover, let $P(t)$ have an antiderivative $P_{1}(t), t \geqslant T$, such that $\lim _{t \rightarrow \infty} P_{1}(t) / t=0$. Then every eventually positive solution $x(t)$ of $(\mathrm{I})$ is increasing and such that $x^{(n-j)}(t)>P(t)$ for all large $t$.

Proof. Let $x(t)$ be a solution of (I) such that $x(t)>0, t \geqslant t_{1} \geqslant T$. Then we have

$$
\left[x^{(n-j)}(t)-P(t)\right]^{(j)}=-H(t, x(t))<0
$$

for every $t \geqslant t_{1}$. Consequently, the function $u(t) \equiv x^{(n-j)}(t)-P(t)$ is either positive or negative for all large $t$. Suppose the latter is true. Then since $j$ is odd, we must also have $u^{\prime}(t)<0$ for all large $t$. Thus, there is $t_{2} \geqslant t_{1}$ such that $u(t)<u\left(t_{2}\right)<0$ for all $t \geqslant t_{2}$. Integrating this last relation we find

$$
x^{(n-j-1)}(t)-P_{1}(t)-\left[x^{(n-j-1)}\left(t_{2}\right)-P_{1}\left(t_{2}\right)\right] \leqslant-u\left(t_{2}\right)\left(t-t_{2}\right)
$$

for every $t \geqslant t_{2}$. This however, along with the fact that $\lim _{t \rightarrow \infty} P_{1}(t) / t=0$, implies $\lim _{t \rightarrow \infty} x^{(n-j-1)}(t)=-\infty$, a contradiction to the positiveness of $x(t)$. Thus, $x^{(n-j)}(t)>P(t) \geqslant 0$ for all large $t$. Since, again, $n-j$ is odd, it follows that $x^{\prime}(t)>0$ for all large $t$, and this completes the proof.

Let us remark now that no conditions were placed on $\left(I_{H}\right)$ concerning its oscillation. Actually, the above theorem concerns itself with the case $x^{(n)}<Q(t)$ because we only used here the sign of $H$. However, its usefulness will become apparent in Theorem 3.4. We present an example just for illustration purposes.

$$
x^{(6)}+t^{-5} x^{3}=-120 t^{-6}+\left[t^{-4} \sin t\right]^{(6)}, \quad t \geqslant 1 .
$$


Here we have $P(t) \equiv 2 t^{-3}+\left[t^{-4} \sin t\right]^{\prime \prime \prime}, \quad P_{i}(t) \equiv-t^{-2}+\left[t^{-4} \sin t\right]^{\prime \prime} . \quad P^{m}(t) \equiv Q(t)$. It follows from the above theorem that every positive solution $x(t)$ of (3.14) satisfies $x^{m}(t)>2 t^{-3}+\varphi(t)$ for all large $t$, where $\varphi(t)=\sigma\left(t^{-4}\right)$.

In the following theorem an even antiderivative of $Q(t)$ is used to ensure upper bounds.

THEOREM 3.3. Let $\left(\mathrm{I}_{H}\right)$ be oscillatory. Moreover, let $P: R_{T} \rightarrow R$ satisfy $P^{(k)}(t) \equiv Q(t)$, where $k$ is an even integer with $2 \leqslant k \leqslant n-2$. Let $\lim _{t \rightarrow \infty} P(t)=0$ and assume the existence of an antiderivative $P_{1}(t)$ of $P(t)$ such that $\lim _{t \rightarrow \infty} P_{1}(t)=\infty$. Then if $x(t)$ is an eventually positive solution of $(\mathrm{I})$, it satisfies $x^{(n-k)}(t)<P(t)$ for all large $t$, and $\lim _{i \rightarrow \infty}\left[x^{(n-k)}(t)-P(t)\right]=0$.

PRoof. Let $x(t)$ be a solution of (I) such that $x(t)>0, t \geqslant t_{1} \geqslant T$. Then as in Theorem 3.2, the function $\mu(t) \equiv x^{(n-k}(t)-P(t), t \geqslant t_{1}$, satisfies $\mu^{(k)}(t)<0$ for all $t \geqslant t_{1}$. Thus $\mu(t)$ is monotonic eventually. Let $\mu(t)>0, t \geqslant t_{2} \geqslant t_{1}$. Then since $k$ is even and $\mu^{(k)}(t)<0$, we must also have $\mu^{\prime}(t)>0$ for all $t \geqslant$ (say) $t_{2}$. Now let $\varepsilon$ be given with $0<\varepsilon<\mu\left(t_{2}\right) / 2$. Then there exists $t_{3} \geqslant t_{2}$ such that $|P(t)|<\varepsilon$ for every $t \geqslant t_{3}$. It follows that

$$
\mu(t)+P(t)>\mu(t)-\varepsilon>\mu\left(t_{2}\right)-\varepsilon>0, \quad t \geqslant t_{3} .
$$

Now integrating the function $\mu(t) \equiv x^{(n-k)}(t)-P(t) n-k$ times we obtain

$$
x(t)=\varphi(t)+\int_{t_{4}}^{t} \int_{t_{4}}^{s_{1}} \ldots \int_{t_{4}}^{s_{n-k-1}}[\mu(s)+P(s)] d s d s_{n-k-1} \ldots d s_{1},
$$

where $t_{4} \geqslant t_{3}$ has been chosen so that $x^{(j)}(t)>0$ for all $t \geqslant t_{4}$ and $0 \leqslant j \leqslant n-k-1$. This is possible because $\lim _{t \rightarrow \infty} P_{1}(t)=+\infty$. In (3.16) $\varphi(t)$ is given by

$$
\varphi(t)=\sum_{j=0}^{n-k-1}\left[\left(t-t_{4}\right)^{j} / j !\right] x^{(j)}\left(t_{4}\right) \geqslant 0, \quad t \geqslant t_{4} .
$$

Thus, $x(t)$ in (3.16) is properly bounded below by the multiple integral for all $t \geqslant t_{4}$. Now inserting $\mu(t)$ in (I) and taking into consideration (3.15) we obtain

$$
\mu^{(k)}(t)+H\left(t, \int_{t_{4}}^{t} \int_{t_{4}}^{s_{1}} \ldots \int_{t_{4}}^{s_{n-k-1}}[\mu(s)-\varepsilon] d s d s_{n-k-1} \ldots d s_{1}\right) \leqslant 0
$$

for all $t \geqslant t_{\mathbf{4}}$. Now let $w(t)$ be the multiple integral above. Then

$$
w^{(n)}(t)+H(t, w(t)) \leqslant 0
$$

with $w(t)$ increasing and positive for every $t \geqslant t_{4}$.

Lemma A, however, implies now the existence of a positive solution of $\left(\mathrm{I}_{H}\right)$, a contradiction. Thus, $x^{(n-k)}(t)-P(t)<0$ for all large $t$. Now let $\lim _{t \rightarrow \infty} \mu(t)=L<0$. Then we can show as in Theorem (3.2) that one integration of $\mu(t)$ and the property $\lim _{t \rightarrow \infty} P_{1}(t) / t=0$ imply the contradiction $\lim _{t \rightarrow \infty} x(t)=-\infty$. Thus $L=0$ and the theorem is proved. 
Let us show now how the lower bounds for the possible eventually positive solutions in Theorem 3.2 can be employed to formulate criteria for the nonexistence of positive solutions.

THEOREM 3.4. Let the assumptions of Theorem 3.2 be satisfied with $P_{1}(t) \rightarrow+\infty$ as $t \rightarrow+\infty$.

Then the conditions

$$
\int_{T}^{t} Q(s) d s \leqslant L, \quad \int_{T}^{\infty} H\left(t, k t^{n-j-1}\right) d t=+\infty
$$

for every $k>0$ and some constant $L$, imply that (I) cannot have an eventually positive solution.

Proof. Let $x(t)$ be a solution of (I) such that $x(t)>0, t \geqslant t_{1} \geqslant T$. Then Theorem 3.2 implies the existence of $t_{2} \geqslant t_{1}$ such that $x^{(n-j)}(t)>P(t)$. Thus,

$$
x^{(n-j-1)}(t)-x^{(n-j-1)}\left(t_{2}\right) \geqslant P_{1}(t)-P_{1}\left(t_{2}\right)
$$

for every $t \geqslant t_{2}$. It follows that given $k>0$, there exists $t_{3} \geqslant t_{2}$ such that $x^{(n-j-1)}(t) \geqslant k$ for every $t \geqslant t_{3}$. Continuing the same way, we obtain $x(t) \geqslant \mu t^{n-j-1}$ for some $\mu>0$ and every $t \geqslant t_{4} \geqslant t_{3}$. Thus from (I) we obtain

$$
\begin{aligned}
x^{(n-1)}(t)-x^{(n-1)}\left(t_{4}\right) & =-\int_{t_{4}}^{t} H(s, x(s)) d s+\int_{t_{4}}^{t} Q(s) d s \\
& \leqslant-\int_{t_{4}}^{t} H\left(s, s^{n-j-1}\right) d s+L_{1},
\end{aligned}
$$

where $L_{1}$ is some positive constant. Obviously, the desired contradiction: $\lim _{t \rightarrow \infty} x(t)=-\infty$ follows from (3.20) by taking limits as $t \rightarrow \infty$.

COROLLARY 3.3. Let the assumption of Theorem 3.2 be satisfied. Moreover, let

$$
\int_{T}^{\infty} H(t, k) d t=+\infty, \quad \int_{T}^{t} Q(s) d s \leqslant L
$$

for some constant $L$ and every $k>0$. Then (I) does not have any positive solution.

The proof follows the steps of Theorem 3.3 with the only difference that $x(t)$ is now bounded below by a positive constant for all large $t$.

COROLlary 3.4. Let the assumptions of Corollary 3.1 and Theorem 3.2 be satisfied with (3.1) oscillatory (B-oscillatory). Then there is no eventually positive (bounded and positive) solution of equation (I).

In fact, Corollary 3.1 ensures that $\lim _{t \rightarrow \infty}$ inf $x(t)=0$ for any eventually positive (bounded and positive) $x(t)$, and Theorem 3.2 says that $x^{(n-j)}(t) \geqslant 0$ eventually. This last inequality implies $x^{\prime}(t) \geqslant 0$ for all large $t$, a contradiction. 
Let us now make the rather interesting remark that if $\lim _{t \rightarrow \infty} P(t)=0$ in Theorem 3.2 , then we do not need to add the hypothesis $\lim _{t \rightarrow \infty} P_{1}(t) / t=0$. In fact, if $u\left(t_{2}\right)<0$ for some $t_{2}$, with $u(t)$ as in the proof of Theorem 3.2, then

$$
x^{(n-j)}(t)-P(t)<u\left(t_{2}\right)
$$

for all $t \geqslant t_{2}$, which implies $x^{(n-j)}(t)<u\left(t_{2}\right)+\varepsilon<0$ for all $t \geqslant$ (some) $t_{3} \geqslant t_{2}$, and some $\varepsilon>0$. This implies again $\lim _{t \rightarrow \infty} x(t)=-\infty$, a contradiction.

Now we apply these considerations to a second-order problem ensuring the nonexistence of positive solutions of the equation

$$
\left[p(t) x^{\prime}\right]^{\prime}+H(t, x)=Q(t) .
$$

This result is related to a theorem of Hammett (1971) and is not contained in any of the results of Atkinson (1972/3).

TheOREM 3.5. For the equation (3.21) assume that $p: \boldsymbol{R}_{T} \rightarrow R_{+} \mid\{0\}$, continuous and such that

$$
\int_{T}^{\infty}[d s / p(s)]=+\infty
$$

Moreover, let for every $k>0$ and $t \geqslant T$,

$$
\int_{T}^{\infty} H(t, k) d t=+\infty, \quad-\int_{1}^{\infty} Q(s) d s>0
$$

where the second integral is assumed to converge.

Then (3.21) does not have any eventually positive solution.

Proof. Let us first note that under the above conditions the homogeneous (3.21) is oscillatory. This follows as in the second theorem of Bhatia (1966). Bhatia assumed that $g^{\prime}(x) \geqslant 0 \dagger$, but this assumption can be dropped by use of suitable Reimann-Stieltjes integrals. Now let the second integral in (3.22) be positive, and let $x(t)$ be a solution of (3.21) such that $x(t)>0, t \geqslant t_{1} \geqslant T$. Then we have

$$
\left[p(t) x^{\prime}(t)+\int_{t}^{\infty} Q(s) d s\right]^{\prime}=-H(t, x(t))<0
$$

for $t \geqslant t_{1}$. Consequently, the function $v(t)$ inside the brackets is decreasing for $t \geqslant t_{1}$. Let $t_{2} \geqslant t_{1}$ be such that $v\left(t_{2}\right)<0$ and let $\varepsilon$ be such that

$$
0<-\int_{l}^{\infty} Q(s d s)<\varepsilon<-v\left(t_{2}\right)
$$

for all $t \geqslant t_{3} \geqslant t_{2}$. Then we have

$$
x^{\prime}(t) \leqslant[v(t)+\varepsilon] / p(t)<\left[v\left(t_{2}\right)+\varepsilon\right] / p(t), \quad t \geqslant t_{3} .
$$

$\dagger$ Bhatia considered the case $H=q(t) g(x)$ with $q(t) \geqslant 0$. 
One integration of (3.24) from $t_{3}$ to $t \geqslant t_{3}$ yields the contradiction $x(t) \rightarrow-\infty$ as $t \rightarrow \infty$. It follows that

$$
x^{\prime}(t)>-[1 / p(t)] \int_{t}^{\infty} Q(s) d s>0, \quad t \geqslant t_{1} .
$$

Thus $x(t)>x\left(t_{1}\right)>0$ for $t \geqslant t_{1}$. Now integrate (3.21) from $t_{1}$ to $t \geqslant t_{1}$ to obtain

$$
p(t) x^{\prime}(t) \leqslant p\left(t_{1}\right) x^{\prime}\left(t_{1}\right)-\int_{t_{1}}^{t} H\left(s, x\left(t_{1}\right)\right) d s+\int_{t_{1}}^{t} Q(s) d s .
$$

Taking limits above as $t \rightarrow \infty$ we obtain $\lim _{t \rightarrow \infty} p(t) x^{\prime}(t)=-\infty$, which leads to a contradiction as in (3.24). A similar proof covers the case of an eventually negative solution, and is therefore omitted.

The proof of the above theorem indicates clearly that its conclusion can be immediately obtained for equations of the form

$$
\left[p_{n-1}(t)\left[\ldots\left[p_{2}(t)\left[p_{1}(t) x^{\prime}\right]^{\prime} \ldots\right]^{\prime}\right]^{\prime}+H(t, x)=Q(t),\right.
$$

where $p_{j}: R_{T} \rightarrow R_{+} \mid\{0\}$, continuous, and such that

$$
\int_{T}^{\infty}\left[d s / p_{j}(s)\right]=+\infty, \quad j=1,2, \ldots, n-1 .
$$

This is actually the reason for presenting its proof because (3.21) can be put back into the form (I) for $n=2$ by letting

$$
\tau=\int_{T}^{\imath} d s / p(s)
$$

\section{Discussion}

It is evident from Theorem 3.1 that a certain amount of information about equation (I) can be obtained from suitable solutions of the inequalities (3.2), (3.3). It turns out that much more information has already been and can be obtained from the study of an $n$th antiderivative of $Q(t)$. However, this simple consideration of a function $P(t)$ with $P^{(n)}(t) \equiv Q(t)$ is balanced in the case of the inequalities (3.2), (3.3) by the fact that in many cases we can exhibit solutions of either one of these inequalities, whose behaviour resembles that of solutions of (I) much more than the behaviour of $P(t)$. Thus, it seems that it is worthwhile looking further into the inequalities (3.2), (3.3).

If we take a closer look at the types of forcings $Q(t)$ in Theorem 3.2 and its corollaries we will realize that $Q(t)$ should be an oscillatory function if $\left(\mathrm{I}_{H}\right)$ is oscillatory. In fact, since $j$ is odd, $Q(t)$ cannot be a positive function. If this was true, then $P(t)$ should be increasing and positive, which contradicts $\lim _{t \rightarrow \infty} P(t)=0$ and $\lim _{t \rightarrow \infty} P_{1}(t) / t=0$. On the other hand, if $Q(t)$ is negative and $\left(\mathrm{I}_{H}\right)$ is oscillatory, then (I) does not have any positive solutions. This follows trivially from Lemma A. 
The $n$th antiderivative of $Q(t)$ was used by Kartsatos $(1971,1972,1975)$ and for $n=2$ by Atkinson (1972/3). It would be very interesting to know any oscillation criteria based on the intermediate antiderivatives of $Q(t)$. It would also be very interesting to obtain some criteria ensuring the monotonicity of all nonoscillatory solutions of (I).

Let us now notice that if there exists a positive function $P(t)$ such that $P^{(n)} \equiv Q(t)$, $t \in R_{T}$, and

$$
P(t)>\int_{T}^{\infty} \int_{u_{n-1}}^{\infty} \ldots \int_{u_{1}}^{\infty} H(s, P(s)) d s d u_{1} \ldots d u_{n-1}
$$

for all $t \geqslant T$, then (I) has at least one positive solution $x(t)$ defined on $[T, \infty)$, and satisfying $x(t)<P(t), t \geqslant T$. This fact follows as in Atkinson (1972/3), Lemma 2, and we omit the proof.

\section{References}

F. V. Atkinson (1972/3), “On second order differential inequalities”, Proc. Royal Soc. Edinburgh, 72, 109-127.

N. P. Bhatia (1966), "Some oscillation theorems for second order differential equations", J. Math. Anal. Appl. 15, 442-446.

K. Foster (1976a), "Oscillations of forced equations", J. Differential Equations 20, 115-132.

K. Foster (1976b), "Oscillations of forced sublinear differential equations of even order", J. Math. Anal. Appl 55, 634-643.

J. R. Graef (to appear), "Oscillation, nonoscillation, and growth of solutions of nonlinear functional differential equations of arbitrary order".

J. R. Graef and P. W. Spikes (to appear), "Asymptotic properties of solutions of functional differential equations of arbitrary order", J. Math. Anal. Appl.

M. E. Hammett (1971), "Nonoscillation properties of a nonlinear differential equation", Proc. AMS, 30, 92-96.

A. G. Kartsatos (1971), "On the maintenance of oscillations of $n$th order equations under the effect of a small forcing term", J. Diff. Equations 10, 355-363.

A. G. Kartsatos (1972), "Maintenance of oscillations under the effect of a periodic forcing term", Proc. $A M S, 34$, 377-383.

A. G. Kartsatos (1975), "On $n$th order differential inequalities", J. Math. Anal. Appl. 52, 1-9.

A. G. Kartsatos (to appear), "Recent results on oscillation of solutions of forced and perturbed nonlinear equations of even order", Proc. NSF-CBMS Reg. Conference, Stability of Dynamical Systems, Mississippi State Univ., Mississippi, edited by J. R. Graef (Marcel Dekker, New York).

A. G. Kartsatos (1976a), "Nth order oscillations with middle terms of order $n-2$ ", Pacific $J$. Math. 67, 477-488.

A. G. Kartsatos (1976b), "Oscillation and existence of unique positive solutions for nonlinear $n$th order equations with forcing term", Hiroshima Math. J. 6, 1-6.

A. G. Kartsatos (1976c), "On the oscillation problem of nonlinear equations", Hiroshima Math. J. 6, 257-263.

A. G. Kartsatos and M. N. Manougian (1974), "Perturbations causing oscillations of functional differential equations", Proc. AMS 43, 111-117.

A. G. Kartsatos and M. N. Manougian (1976), "Further results on oscillation of functional differential equations", J. Math. Anal. Appl. 53, 28-37. 
T. Kusano and H. Onose (1974), "Oscillations of functional differential equations with retarded argument", J. Diff. Equations 15, 269-277.

Z. Nehari (1963), "On a nonlinear differential equation arising in nuclear physics", Proc. Royal Irish Acad. 62, 117-135.

H. Onose (1973), "Oscillation and asymptotic behaviour of solutions of retarded differential equations of arbitrary order", Hiroshima Math. J. 3, 333-360.

H. Onose (1975), "A comparison theorem and the forced oscillation", Bull. Austral. Math. Soc. 13, 13-19.

V. A. Staikos and Y. G. Sficas (1975a), "Forced oscillation for differential equations of arbitrary order", J. Diff. Equations 17, 1-11.

V. A. Staikos and Y. G. Sficas (1975b), "Oscillations for forced second order nonlinear differential equations", Atti Acad. Naz. Lincei Rend. Cl. Sci. Fis. Mat. Natur. 58, 318-322.

H. Teufel, Jr. (1972), "Forced second order nonlinear oscillation", J. Math. Anal. Appl. 40, 148-152.

E. True (1975), "A comparison theorem for certain functional differential equations", Proc. AMS, 47, 127-132.

\section{Department of Mathematics}

University of South Florida

Tampa Florida 33620

USA 